\title{
Prehospital triage accuracy in a criteria based dispatch centre
}

Fabrice Dami ${ }^{1,2^{*}+}$, Christel Golay ${ }^{3^{*+}}$, Mathieu Pasquier ${ }^{2}$, Vincent Fuchs ${ }^{1}$, Pierre-Nicolas Carron ${ }^{2}$ and Olivier Hugli ${ }^{2}$

\begin{abstract}
Background: Priority dispatch accuracy is a key issue in optimizing the match between patients' medical needs and pre-hospital resources. This study measures the accuracy of a Criteria Based Dispatch (CBD) system, by evaluating discrepancies between dispatch priorities and ambulance crews' severity evaluations.

Methods: This is a retrospective study conducted from January 2011 to December 2011. We ruled that a National Advisory Committee for Aeronautics (NACA) score $>3$ (injuries/diseases which can possibly lead to deterioration of vital signs) to 7 (lethal injuries/ diseases) should require a priority dispatch with lights and siren (L\&S), while NACA scores $<4$ should require a priority dispatch without L\&S. Over triage was defined as the proportion of L\&S dispatches with a NACA score $<4$, and under triage as the proportion of dispatches without L\&S with a NACA score $>3$.
\end{abstract}

Results: There were 29,008 primary missions in 2011, 1122 were excluded. Of the 15,749 L\&S missions, 12,333 patients had a NACA score < 4, leading to an over triage rate of $78 \%$; 561 missions out of 12,137 missions without L\&S had a NACA score > 3, leading to an under triage rate of $4.6 \%$. Sensitivity was $86 \%$ (95\% confidence interval: $85.6-86.4 \%)$, specificity $48 \%$ (47.4-48.6\%), positive predictive value $21.7 \%$ (21.2-22.2\%), and negative predictive value $95.4 \%$ (95.2-95.6\%).

Conclusion: The rates of over triage and under triage in our CBD are 78 and $4.6 \%$ respectively. The lack of consistent or universal metrics is perhaps the most important limitation in dispatch accuracy research. This is mainly due to the large heterogeneity of dispatch systems and prehospital emergency system.

Keywords: EMS, Criteria-based dispatch, Accuracy, Benchmarking

\section{Background}

Priority dispatch accuracy is a key issue in optimizing the match between patients' medical needs and prehospital resources [1]. Although it is less studied than early cardiac arrest identification and telephone-CPR instructions, this topic is a major issue for dispatch centres as they all try to achieve the most efficient use of their resources. Over triage from dispatch centres represents an immediate response with lights and sirens (L\&S) for a low-acuity case. It consumes limited resources, may increase costs and causes a shortage of ambulances for

\footnotetext{
*Correspondence: Fabrice.dami@chuv.ch; Christel.golay@unil.ch ${ }^{\dagger}$ Equal contributors

'Dispatch centre, State of Vaud (Fondation Urgences-Santé), César-Roux 31, 1005 Lausanne, Switzerland

${ }^{3}$ Faculty of Biology and Medicine, University of Lausanne, Lausanne, Switzerland

Full list of author information is available at the end of the article
}

high-acuity emergencies; it could also endanger emergency medical services (EMS) workers and the general population, with ambulances running hot [2] with no or little benefit to the patient [3]. On the other hand, under triage from dispatch centres represents an inappropriately low response without priority signs in the presence of an acute case. Although this has not been documented at the dispatch level, it may place the patients at risk of transient unmet medical needs and delayed access to the appropriate level of care as it is for trauma patients from field triage [4].

Today there is no consensus on the accepted percentage of over and under triage at the dispatch level because of a very high heterogeneity in EMS. Different types of dispatch systems are used within the world: medical priority dispatch system (MPDS), which is the most widespread, is mainly found in North America $[5,6]$ and in the

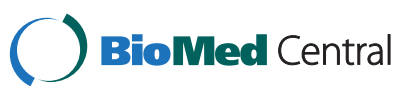

(c) 2015 dami et al. Open Access This article is distributed under the terms of the Creative Commons Attribution 4.0 International License (http://creativecommons.org/licenses/by/4.0/), which permits unrestricted use, distribution, and reproduction in any medium, provided you give appropriate credit to the original author(s) and the source, provide a link to the Creative Commons license, and indicate if changes were made. The Creative Commons Public Domain Dedication waiver (http://creativecommons.org/publicdomain/zero/1.0/) applies to the data made available in this article, unless otherwise stated. 
UK [7]; Criteria Based Dispatch (CBD) systems are almost exclusively located in European countries (including Denmark [8], Norway [9], Belgium [10], and regions of Switzerland [11]); physician dispatch is used in France [12]. There are also different types of resources available within the world of Emergency Medical Services (EMS): presence of advanced life support teams and/or basic life support teams, with or without first-responders or prehospital emergency physicians, which may influence dispatch protocols.

Our hypothesis is our dispatch has a high level of overtriage. The aim of this study was to evaluate the accuracy of a CBD system, by evaluating discrepancies between the dispatch priorities and ambulance crews' severity evaluations, and to quantify the over and under triage.

\section{Methods}

\section{Setting}

This study was conducted throughout the State of Vaud in the French-speaking region of Switzerland, where a centralized pre-hospital medical dispatch centre serves a population of 750,000 and handles over 80,000 calls per year. The dispatch centre is staffed by registered nurses and certified paramedics with at least 5 years of field experience. It is a CBD system based on caller's description of symptoms. All calls answered are assigned a keyword from a pre-determined list. Not only must dispatchers categorize every call, it is also mandatory for them to inquire on the victim's 'state of consciousness' and 'quality of breathing' to detect cardiac arrest. Dispatchers rely on their own medical background and personal experience to ask the questions they deem appropriate to perform the interview. Each call is processed by the same dispatcher from the beginning (interview) to the end (dispatch). When appropriate, they deliver telephone-guided life-saving maneuvers to bystanders [13]. They benefit from $40 \mathrm{~h}$ of continuing education every year and are regularly evaluated to ensure that our quality standards are met. In Switzerland, priority 1 (immediate departure with $L \& S$ ) is required only if dispatchers believe that there is a vital risk for the patient. Priority 2 is an immediate departure without L\&S and priority 3 is a delayed departure [14]. Ambulances assigned a priority level 1 or 2 are staffed with at least one paramedic, while ambulances assigned a priority level 3 may be staffed with emergency medical technicians only.

Our pre-hospital network is a three-tier system. Ambulance crews dispose of state protocols for autonomous intravenous access, cardiopulmonary resuscitation procedures, defibrillation, and emergency medication administration [15]. Pre-hospital emergency physicians may be dispatched to the site by the call centre or later at the request from paramedics, either by ground or by helicopter.

When dealing with a low-acuity case that may not need a transport, our dispatchers can transfer the caller to the state's nurse-counselling dispatch which provides medical advice or can dispatch an on-call general practician 24/7 within an hour. Those cases are not included in this study, unless the on-call physician decided a transport was necessary after visiting the patient.

Most of the scheduled and non-urgent transports from nursing homes to the hospitals or between hospitals do not undergo an evaluation by our dispatch centre as they are directly organized by the nursing home or hospital with the ambulance company. Those cases are not included in this study.

\section{Study design}

We retrospectively studied the registry database of our dispatch centre, from January $1^{\text {st }} 2011$ to December $31^{\text {st }}$ 2011. The registry records all dispatches in our State; pre-hospital teams input their data at the end of each mission. We excluded secondary missions (inter-hospital transfers), helicopter missions without ambulance already on site, missions aborted, and those with missing data.

The data collected from each mission were the priority chosen by dispatcher, the keyword or determinant chosen to qualify the situation, and the severity of the condition assessed by the pre-hospital crews according to the National Advisory Committee for Aeronautics (NACA) score and transmitted to the dispatch at the end of the mission [16, 17]. The NACA score is an eight-level scale to assess pre-hospital severity status; the score is defined by the most serious clinical state experienced at any given time during the mission. (Fig. 1) The NACA score is commonly used in western Europe in pre-hospital emergency medicine and is significantly correlated with survival $[18,19]$. The NACA score enables categorization of the victims' condition, allows for statistical reviews of the type of injuries and illnesses treated, and illustrates the case-mix of pre-hospital health-care professionals. This scoring is mandatory in Switzerland for all ambulance and helicopter missions.

We initially ruled that a NACA score of 4 (injuries/ diseases which can possibly lead to deterioration of vital signs) to 7 (lethal injuries or diseases, with or without resuscitation attempts) should require a P1 dispatch priority (with $L \& S$ ), while NACA scores under 4 should require a P2 or P3 dispatch priority (without L\&S). Over triage was defined as the proportion of $\mathrm{P} 1$ dispatches for patients with NACA score $<4$, and under triage as the proportion of P2 and P3 dispatches for patients with NACA score $>3$. The main outcome of the study was to measure the correlation between priority dispatch 
NACA O No injury or disease

NACA 1 Injuries/diseases without any need for acute physicians care

NACA 2 Injuries/diseases requiring examination and therapy by a physician but hospital admission is not indicated. Including: large contusions, finger and toe fracture, $2^{\text {nd }}$ degree burn (10-20\% of body surface), exhaustion without hypothermia

NACA 3 Injuries/diseases without acute threat to life but requiring hospital admission. Including: maxillofacial trauma, wound with vascular/neurological impact, $3^{\text {rd }}$ degree burn (10-20\%), hypoglycemia without coma, TIA, supra-ventricular arrhythmia with conserved hemodynamic, right iliac fossa pain syndrome, hypothermia stage I, $2^{\text {nd }}$ degree burn (20$30 \%$ ), isolated limb fracture (femur excluded)

NACA 4 Injuries/diseases which can possibly lead to deterioration of vital signs. Including: open skull fracture, hypothermia stage II, suspicion SCA, suspicion ectopic pregnancy/placenta praevia

NACA 5 Injuries/diseases with acute threat to life. Including: head trauma GCS<8, heart infarct, bradycardia (<30/min), tachycardia (>180/min), complete heart bloc, eclampsia, hypothermia stage III, haemodynamic shock, multiple ribs fractures, acute dyspnea, pulmonary edema

NACA 6 Injuries/diseases transported after successful resuscitation. Including: chest trauma with severe dyspnea, aortic rupture, airways total obstruction, central apnea, emergency external pacing, cardiac arrest (ventricular fibrillation or asystole from any cause)

NACA 7 Lethal injuries or diseases (with or without resuscitation attempts)

Fig. 1 National Advisory Committee for Aeronautics (NACA) score revised by the State of Vaud, Switzerland (2005)

assigned by the dispatcher (P1-P2-P3) and the NACA score given by EMS personnel. The score given by the EMS personnel was considered the gold standard and therefore defined over and under triage by the dispatcher.

\section{Statistics}

Simple descriptive statistics were used. Sensitivity, specificity, positive and negative predictive values, averages, and percentages were calculated with Microsoft Office Excel 2007.

\section{Results}

There were 29,008 primary missions in 2011 of which 1122 were excluded, leaving 27,886 missions included in our study: 15,749 P1 (57 \%), 8484 P2 (30 \%), 3653 P3 (13\%) (Fig. 2).

Of the $15,749 \mathrm{P} 1$ missions 12,333 patients had a NACA score $<4$, leading to a rate of over triage of $78 \%$; 561 missions out of 12,137 P2/P3 missions had a NACA score $>3$, leading to an under triage of $4.6 \%$. Sensitivity of our triage was $86 \%$ (95\% confidence interval: 85.6-86.4\%), specificity $48 \%$ (47.4-48.6\%) while positive predictive value (PPV) was $21.7 \%(21.2-22.2 \%)$ and negative predictive value (NPV) 95.4 \% (95.2-95.6\%) (Table 1).

The most frequent scores attributed at the end of the missions were NACA 2 and 3 (54.5\% and $25.6 \%$, respectively). There was a majority of P1 dispatch in all NACA categories (mean: $56.5 \%$ ), ranging from $46.2 \%$ for NACA 3 to $98.1 \%$ for NACA 7. According to our definition of concordance, dispatcher and EMS agreed on priority in $53.8 \%$ of missions (P1 for NACA score $>3$ and P2/P3 for NACA score <4). High-acuity cases (NACA >3) represented $14 \%$ of all missions (Table 1). Paediatric cases (<18 years old) represented only $5 \%$ of our case mix. Table 2 resumes the use of dispatch keywords in 2011. "Undefined problem" represents $60 \%$ of our case-mix.

Most of the over triage concerns NACA 3 (57\%) and keywords 'undefined problem' (4676; 38 \%), 'public place' (2847; $23 \%)$, 'disturbance of consciousness' (1353; $11 \%)$, 


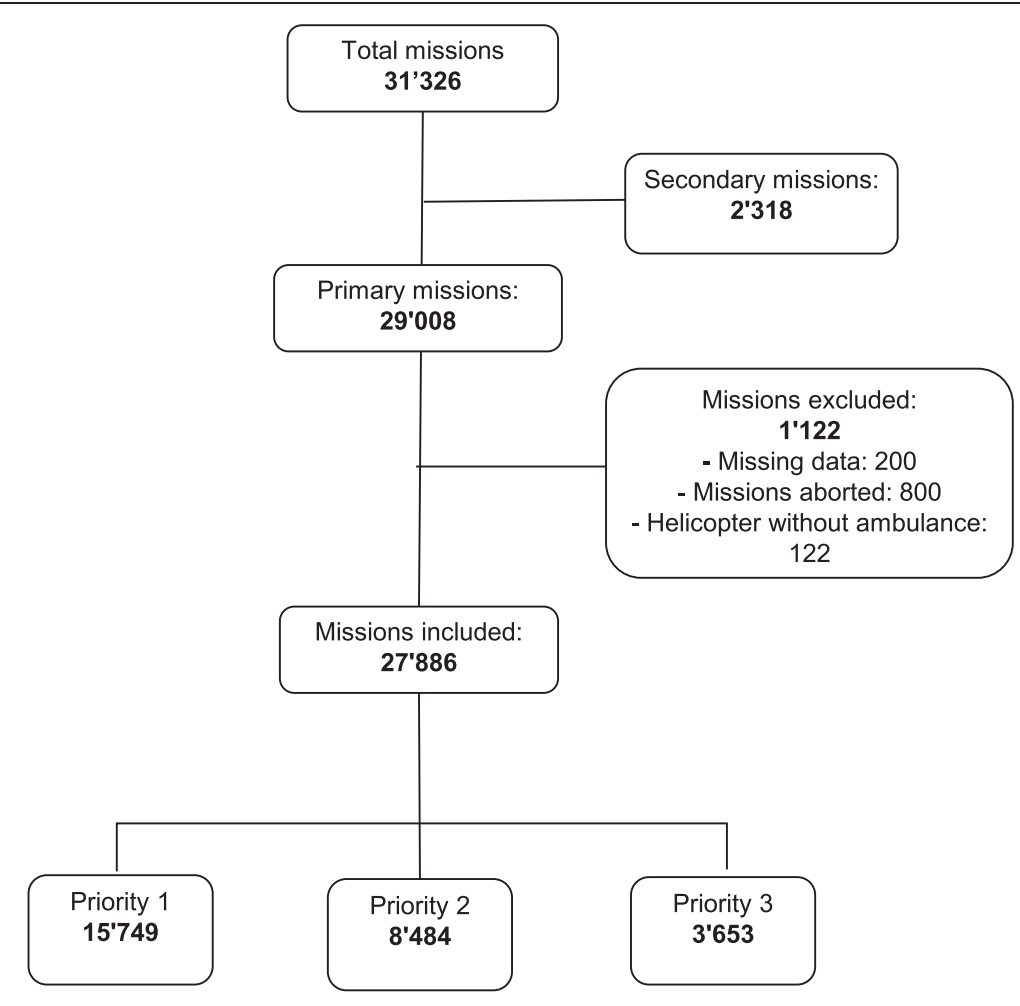

Priority 1 dispatch: immediate departure with lights and siren

Priority 2 dispatch: immediate departure without lights and siren

Priority 3: delayed departure without ligths and siren

Fig. 2 Flow chart

Table 1 Priority dispatch and NACA score

\begin{tabular}{llllll}
\hline Missions & Total $[n(\%)]$ & $P 1[n(\%)]$ & $P 2[n(\%)]$ & $P 3[n(\%)]$ & Correct correlation $[n(\%)]$ \\
\hline NACA 0 & $332(1.2)$ & $184(55.4)$ & $128(38.6)$ & $20(6.0)$ & $148(44.6)$ \\
NACA 1 & $1^{\prime} 247(4.5)$ & $830(66.5)$ & $371(29.8)$ & $46(3.7)$ & $417(33.4)$ \\
NACA 2 & $7^{\prime} 122(25.6)$ & $4^{\prime} 285(60.2)$ & $2^{\prime} 228(31.3)$ & $609(8.5)$ & $2^{\prime} 837(39.8)$ \\
NACA 3 & $15^{\prime} 208(54.5)$ & $7^{\prime} 034(46.2)$ & $5^{\prime} 290(34.8)$ & $2^{\prime} 884(19.0)$ & $8^{\prime} 174(53.7)$ \\
NACA 4 & $2^{\prime} 480(8.9)$ & $2^{\prime} 034(82.0)$ & $376(15.2)$ & $70(2.8)$ & $22^{\prime} 034(82.0)$ \\
NACA 5 & $867(3.1)$ & $767(88.5)$ & $79(9.1)$ & $21(2.4)$ & $767(88.5)$ \\
NACA 6 & $203(0.7)$ & $196(96.5)$ & $5(2.5)$ & $2(1.0)$ & $196(96.6)$ \\
NACA 7 & $427(1.5)$ & $419(98.1)$ & $7(1.7)$ & $1(0.2)$ & $419(98.1)$ \\
Total & $27^{\prime} 886(100)$ & $15^{\prime} 749(56.5)$ & $8^{\prime} 484(30.4)$ & $3^{\prime} 653(13.1)$ & $14^{\prime} 992(53.8)$ \\
\hline
\end{tabular}

OVER TRIAGE $=$ P1 dispatch with NACA $<4$ (false positive) $/$ all P1 dispatch (false positive + true positive) $=12^{\prime} 333 / 15^{\prime} 749=78 \%$

UNDER TRIAGE $=$ P2 or P3 dispatch with NACA $>3$ (false negative) $/$ all P2 or P3 dispatch (false negative + true negative) $=561 / 12^{\prime} 137=4.6 \%$

Sensitivity was calculated as true positives/(true positives + false negatives); specificity, as true negatives/(false positives + true negatives)

Positive predictive value (PPV) was calculated as true positives/(true positives + false positives); negative predictive value (NPV), as true negatives/(true negatives + false negatives)

Sensibility $=\mathrm{TP} /(\mathrm{TP}+\mathrm{FN})=86 \%=95 \% \mathrm{Cl}(85.6-86.4)$

Specificity $=\mathrm{FN} /(\mathrm{FN}+\mathrm{FP})=48 \%=95 \% \mathrm{Cl}(47.4-48.6)$

$\mathrm{PPV}=\mathrm{TP} /(\mathrm{TP}+\mathrm{FP})=21.7 \%=95 \% \mathrm{Cl}(21.2-22.2)$

$\mathrm{NPV}=\mathrm{TN} /(\mathrm{TN}+\mathrm{FN})=95.4 \%=95 \% \mathrm{Cl}(95.2-95.6)$ 
Table 2 Keywords used in 2011

\begin{tabular}{|c|c|}
\hline Keyword & Missions $[n(\%)]$ \\
\hline Undefined problem & $16^{\prime} 739(60.1)$ \\
\hline Public place & $3255(11.7)$ \\
\hline Dyspnoea & 1'807 (6.4) \\
\hline Disturbance of consciousness & $1^{\prime} 768(6.3)$ \\
\hline Chest pain & $1^{\prime} 449(5.2)$ \\
\hline Unconscious & 1'364 (4.9) \\
\hline Acute stroke $<5 \mathrm{~h}$ & $428(1.5)$ \\
\hline Child (accident) & $373(1.3)$ \\
\hline Intoxication & $169(0.6)$ \\
\hline Child (dyspnoea) & $98(0.4)$ \\
\hline Hypotension (symptomatic) & $70(0.3)$ \\
\hline Fall above $3 \mathrm{~m}$ & $60(0.2)$ \\
\hline Penetrating injury & $46(0.2)$ \\
\hline Delivery (imminent) & $45(0.2)$ \\
\hline Incarcerated & $42(0.2)$ \\
\hline Prevention (fire) & $35(0.1)$ \\
\hline Fall above $5 \mathrm{~m}$ & $34(0.1)$ \\
\hline Impossible access for ambulance (mountains) & $25(0.1)$ \\
\hline Demand from on-call physician & $15(0.1)$ \\
\hline Burns (limited) & $14(0.1)$ \\
\hline Child (unconscious) & $9(0.0)$ \\
\hline Limb's amputation & $8(0.0)$ \\
\hline Burns (extensive, >10 \%) & $6(0.0)$ \\
\hline Drowning & $6(0.0)$ \\
\hline Ejected & $5(0.0)$ \\
\hline Para/tetraplegia & $4(0.0)$ \\
\hline Car accident (>3 patients) & $3(0.0)$ \\
\hline Explosion & $2(0.0)$ \\
\hline Electrocution & $2(0.0)$ \\
\hline Anaphylactic reaction & $2(0.0)$ \\
\hline Diving accident & $2(0.0)$ \\
\hline Accident in a tunnel & $1(0.0)$ \\
\hline TOTAL & $27^{\prime} 886(100)$ \\
\hline
\end{tabular}

'chest pain' (947; $8 \%$ ), 'dyspnoea' (894; $7 \%$ ), 'unconscious' (676; $6 \%$ ) (Table 3).

Most of the under triage concerns NACA 4 (79.5\%) with keywords 'undefined problem' (469; $83.6 \%$ ), 'dyspnoea' (54; $9.6 \%)$ and 'disturbance of consciousness' (21; $3.7 \%)$ (Table 4).

\section{Discussion}

Our rate of over triage is $78 \%$ and our rate of under triage is $4.6 \%$. There are published recommendation rates of over and under triage in pre-hospital trauma field medicine by the American College of Surgeons
Committee on Trauma (5-10\% of under triage and $30-50 \%$ of over triage) [20], but we are still missing such consensus on acceptable rates and objectives in medical dispatch. Thirty-one experts met in 2004 to establish standards on EMS studies, but specifically no agreement was found on over and under triage in particular [21].

Some looked for concordance between dispatch priorities and emergency department (ED) evaluation [22], or between dispatch, EMS and ED [23]. We decided to compare dispatch priorities and EMS field findings only, as EDs' evaluation cannot take into account any significant change in the patient's condition due to time and pre-hospital intervention. Priority dispatch should be evaluated by the first professional on site, so the impact of elapsed time from dispatch to clinical evaluation is minimal. Treatments provided by EMS may contribute to the improvement of the patient's clinical condition, which will also interfere with the evaluation by ED personal of dispatch priority.

Benchmarking remains difficult even when looking only at studies that did compare dispatch priorities and EMS findings rather than ED evaluations. Dispatch centres (CBD, MPDS, physician dispatch) and EMS systems (two or three tiers) are very heterogeneous. Although all previous studies have dealt with the same metric issue (high versus low-acuity cases) there is no consensus on their definition. Therefore criteria to define a concordance between dispatch priority and the clinical findings on the field by EMS (gold standard) differ widely.

Table 5 presents results from previous studies dispatch over and under triage. Those examples show that not only are dispatch criteria different between studies, but also case acuity measurement tools by on field EMS. Therefore it is not possible to conclude that one system may be more efficient than another, whatever rates of over and under triage are published. Nevertheless, it is of prime importance for dispatch centres to publish their results as this may allow benchmarking and, with time, permit the reaching of an international consensus on dispatch accuracy.

Scott et al. remind us that during the seventies, studies showed that the outcome of non-traumatic cardiac arrests was linked to rapid EMS dispatch $[24,25]$. This observation paved the way to the 'eight-minutes response' that all dispatch centres within United States try to achieve for Advanced Life Support interventions. However, according to a 2002 systematic review [26], and to Snooks et al. when defining the highest priorities for research in emergency pre-hospital care in 2009, there is still insufficient evidence to generalize the effect of the prioritization of emergency ambulances on patient 
Table 3 Over triage by keywords

\begin{tabular}{|c|c|c|c|c|c|}
\hline & Total $[n(\%)]$ & $\operatorname{NACA} 0[n(\%)]$ & NACA $1[n(\%)]$ & NACA $2[n(\%)]$ & NACA $3[n(\%)]$ \\
\hline $\mathrm{N}$ (P1 over triage) & $12^{\prime} 333(100)$ & $184(1.5)$ & $830(6.7)$ & $4^{\prime} 285(34.8)$ & $77^{\prime} 034(57.0)$ \\
\hline Undefined problem & $4^{\prime} 676(37.9)$ & 71 & 325 & $1^{\prime} 640$ & $2^{\prime} 640$ \\
\hline Public place & $2^{\prime} 847(23.1)$ & 53 & 226 & $1^{\prime} 289$ & $1^{\prime} 279$ \\
\hline Disturbance of consciousness & 1'353 (11.0) & 24 & 93 & 407 & 829 \\
\hline Chest pain & $947(7.7)$ & 4 & 26 & 220 & 697 \\
\hline Dyspnoea & $894(7.3)$ & 11 & 67 & 243 & 573 \\
\hline Unconscious & $676(5.4)$ & 14 & 45 & 176 & 441 \\
\hline Demand from on-call physician & $247(2.0)$ & 1 & 5 & 45 & 196 \\
\hline Others & 693 (5.6) & 6 & 43 & 265 & 379 \\
\hline
\end{tabular}

outcome other than cardiac arrest, and therefore it may not be a useful indicator [1, 27].

The future of dispatching may rely on a very structured interview based on questions with high PPV to avoid under triage, or NPV to avoid over triage for lifethreatening conditions, combined with sophisticated computer algorithms to estimate better the patient's life threat risk [28]. In a study from Japan, this system resulted in an over triage's rate of $35 \%$ and an under triage of less than $1 \%$ for cases when looking specifically at cardio-pulmonary arrest cases (sensitivity $80.2 \%$ (95 \% CI: 78.6-81.8\%), specificity $96.0 \%$ (95\% CI: 95.8-96.1 \%), PPV $42.6 \%$ (95 \% CI: 41.1-44.0\%), and NPV 99.2 \% (95 \% CI: 99.2-99.3\%) respectively) [29].

\section{Over triage (Table 3)}

'Undefined problem', which represents $38 \%$ of over triage, can be used when the dispatcher did not get enough information from the caller, either because he/she did not ask the necessary questions, or because the caller was not near the patient or was too agitated to provide meaningful answers. It may also be used when a serious condition is suspected and dispatchers tend to quickly dispatch without paying too much attention to the categorization of the call. This keyword was replaced in our dispatch in 2012 by 'disease not classified elsewhere' and 'trauma not classified elsewhere' to slightly improve the description of the case, and therefore the quality of our case mix.

'Public place' represents $23 \%$ of over triage. Historically P1 dispatch was required by the police department on those cases for security reasons. Since 2012, however, this has been suppressed to respect the classical dispatch criteria (mechanism, interview, clinical signs).

“Disturbance of consciousness' represents $11 \%$ of over triage. This keyword is associated with over triage $77 \%$ of the time. Regarding the large spectra of non-specific problems that this term encompasses (orthostatism, alcohol or drug abuse, arrhythmia), its performance may be difficult to improve. 'Dyspnoea' and 'chest pain' represent $7 \%$ and $8 \%$ of over triage respectively. Finally, there were 247 cases for which P1 was requested by an on-call physician on site but that were classified with NACA score $\leq 3$. Dispatchers are therefore not responsible for those cases of over triage.

We did not evaluate over triage between P2 and P3 priorities, as there is no official clinical criterion to discriminate these two dispatch levels. This choice mainly relies on the dispatcher's clinical judgement. Furthermore we have not been able to set a standard to discriminate those priority levels as we did to discriminate $\mathrm{P} 1$ vs $\mathrm{P} 2$ and P3.

Table 4 Under triage by keywords

\begin{tabular}{|c|c|c|c|c|c|}
\hline & Total [n (\%)] & NACA 4 [n(\%)] & NACA 5 [n(\%)] & NACA 6 [n(\%)] & NACA 7 [n(\%)] \\
\hline$N(P 2+P 3$ under triage) & $561(100)$ & $446(79.5)$ & $100(17.8)$ & $7(1.3)$ & $8(1.4)$ \\
\hline Undefined problem & 469 (83.6) & 377 & 80 & 7 & 5 \\
\hline Dyspnoea & $54(9.6)$ & 40 & 14 & 0 & 0 \\
\hline Disturbance of consciousness & $21(3.7)$ & 14 & 6 & 0 & 1 \\
\hline Chest pain & $7(1.2)$ & 7 & 0 & 0 & 0 \\
\hline Public place & $4(0.7)$ & 4 & 0 & 0 & 0 \\
\hline Acute stroke & $2(0.4)$ & 2 & 0 & 0 & 0 \\
\hline Intoxication & $2(0.4)$ & 2 & 0 & 0 & 0 \\
\hline Prevention & $2(0.4)$ & 0 & 0 & 0 & 2 \\
\hline
\end{tabular}


Table 5 Previous results on dispatch over and under triage

\begin{tabular}{|c|c|c|c|c|c|c|}
\hline & Over triage & Under triage & Sensitivity & Specificity & PPV & NPV \\
\hline \multicolumn{7}{|l|}{ Criteria Based Dispatch } \\
\hline Korram-Manesh A, et al. [23] & $73 \%$ & $3.5 \%$ & NA & NA & NA & NA \\
\hline Ek B, et al. [33] & NA & NA & $95.9 \%$ & $15.4 \%$ & $88.5 \%$ & $29.1 \%$ \\
\hline \multicolumn{7}{|l|}{ Medical Priority Dispatch } \\
\hline Lu TC, et al. [34] & $62.9 \%$ & $8.1 \%$ & NA & NA & NA & NA \\
\hline Sporer KA, et al. [35] & NA & NA & $84 \%$ & $36 \%$ & $84 \%$ & $35 \%$ \\
\hline Feldman MJ, et al. [6] & $34 \%$ & $32 \%$ & $68.2 \%$ & $66.2 \%$ & $80.3 \%$ & $50.7 \%$ \\
\hline Neely KW, et al. [36] & $29 \%$ & $5.4 \%$ & NA & NA & NA & NA \\
\hline
\end{tabular}

Seventy-eight per cent of over triage is not an acceptable rate and this needs to be improved.

\section{Under triage (Table 4)}

With 446 missions, NACA 4 missions with the keywords 'undefined problem' (83.6 \%) and dyspnoea' (9.6\%) were the major sources of under triage. There are 36 cases (NACA >3) with on-call physician specifically not asking for a P1, most of them related to palliative care, which should not be considered as dispatch under triage. There are 8 NACA 7 cases that did not receive a P1 dispatch. This is due to voluntarily under triage by dispatchers when they believe death is certain or when palliative care is appropriate regarding information transmitted from the caller (spouse, nurse, doctor).

Although it has not been specifically measured in our work, we suggest that palliative care cases should be excluded from those studies if, at the time of the call, clinical instability is present but the level of priority dispatch chosen is low because of the patient's choice or the decision by the physician on site.

It has not been demonstrated that under triage has any impact on patients' outcome. "An accepted hierarchy of time-dependent interventions and thresholds for under triage is necessary for the judicious analysis and optimal design of a tiered EMS system" [5].

The ideal definition of under triage would be when an inappropriate priority level answer had an impact on the patient's outcome. But this often remains subjective and therefore difficult to measure.

A first step allowing benchmarking between different EMS regarding over and under triage, would be to propose a list of clinical findings deserving dispatch with L\&S. As all dispatch use L\&S for high acuity case, this may allow the correlation of the perceived acuity by the dispatch centre with the clinical's acuity on site, whatever dispatch system is used and whatever level of competences is available on board ambulances.

\section{Limitations}

This is an observational retrospective study in a specific setting, not applicable to other dispatch systems or twotier EMS.

We used the NACA score to evaluate the patient's severity status, as this score has been significantly correlated with short-term survival, transfer to the intensive care unit and hospital length of stay [17-19] although it has been described as partly subjective [30] and not always reproducible [31]. The NACA score defines the most serious clinical state experienced at any given time during the mission. Therefore it may not always describe the patient's clinical state on EMS arrival, as a minority of patients worsened during EMS care or transport, which may overestimate undertriage. A clinical evaluation on EMS arrival would be the best tool to evaluate dispatch's decision.

All studies comparing a patient's clinical condition at two different times (at time of dispatch and EMS clinical evaluation in this study) are subject to a limitation, as the patient's situation can change for better or worse while EMS is on its way. Therefore, differences between the two time points may reflect an incorrect assessment by the dispatcher, or a change in the patient's condition.

'Undefined problem' is the most used keyword in our dispatch in 2011. The overuse of this code (60\% of all missions), a much higher rate than in previous studies, lowers the quality of our case-mix description and illustrates the difficulties in describing and assessing emergency calls $[8,32]$.

\section{Conclusion}

The rates of over triage and under triage in our CBD are 78 and $4.6 \%$ respectively. The common estimation of high-acuity cases in the literature is $10 \%$ of all calls, $14 \%$ in our work. All centres should tend to achieve that proportion of high-acuity dispatches with the highest sensitivity and specificity possible, keeping in mind triage sensitivity and specificity are inversely related. The lack of consistent or universal metrics for the conduct of 
studies is perhaps the most important limitation in dispatch accuracy research. This is mainly due to the large heterogeneity of dispatch systems and competences of ambulance crews. A consensus on cases deserving dispatch with $L \& S$ could be a first step allowing benchmarking regarding over and under triage by dispatch centres.

\section{Ethics committee}

This study was authorized by the Lausanne University Ethics Committee for human research.

\section{Abbreviations}

CBD: Criteria based dispatch; ED: Emergency Department; EMS: Emergency medical services; L\&S: Lights and siren; MPDS: Medical priority dispatch system; NACA: National advisory committee for aeronautics; NPV: Negative predictive value; PPV: Positive predictive value.

\section{Competing interests}

FD, main investigator, is employed part-time as the EMS Medical Director and part-time as senior physician at the Emergency Department of the University Hospital of Lausanne (CHUV). There are no other competing interests and sources of funding.

\section{Authors' contribution}

FD and CG conceived the study, analysed the data and equally worked on the manuscript. MP and PNC participate in its design. VF and MP helped to conceive the study and analyse the data. $\mathrm{OH}$ helped to draft the manuscript. All authors read and approved the final manuscript.

\section{Acknowledgements}

We would like to thank our dispatchers for their professionalism and ability to manage stressful situations, as well as for participating in our study to improve the efficiency of the chain of survival.

\section{Author details}

'Dispatch centre, State of Vaud (Fondation Urgences-Santé), César-Roux 31, 1005 Lausanne, Switzerland. ${ }^{2}$ Department of Emergency Medicine, University Hospital Center (CHUV), Bugnon 46, 1011 Lausanne, Switzerland. ${ }^{3}$ Faculty of Biology and Medicine, University of Lausanne, Lausanne, Switzerland.

Received: 3 June 2015 Accepted: 19 October 2015

Published online: 27 October 2015

\section{References}

1. Snooks H, Evans A, Wells B, Peconi J, Thomas M, Woollard M, et al. What are the highest priorities for research in emergency prehospital care? Emerg Med J. 2009;26:549-50.

2. Maguire JB, Hunting KL, Smith GS, Levick NR. Occupational fatalities in EMS: a hidden crisis. Ann Emerg Med. 2002;40:625-32.

3. Merlin M, Baldino KT, Lehrfeld DP, Linger M, Lustiger E, Cascio A, et al. Use of a limited lights and siren protocol in the prehospital setting vs. standard usage. Am J Emerg Med. 2012;30:519-25.

4. Mackenzie EJ, Rivara FP, Jurkovich GJ, Nathens AB, Frey KP, Egleston BL, et al. A national evaluation of the effect of trauma-center care on mortality. NEJM. 2006;354:366-78.

5. Sporer KA, Johnson NJ, Clement CY, Youngblood GM. Can emergency medical dispatch codes predict prehospital interventions for common 9-1-1 call types? Prehosp Emerg Care. 2008;12:470-8.

6. Feldman MJ, Verbeek PR, Lyons DG, Chad SJ, Craig AM, Schwartz B. Comparison of the medical priority dispatch system to an out-of-hospital patient acuity score. Acad Emerg Med. 2006;13:954-60.

7. Clegg GR, Lyon RM, James S, Branigan HP, Bard EG, Dispatch-assisted $E G J$, et al. Where are the hold-ups during calls to emergency dispatchers? a preliminary analysis of caller-dispatcher interactions during out-of-hospital cardiac arrest using a novel call transcription technique. Resuscitation. 2014;85:49-52.
8. Andersen MS, Johnsen SP, Sørensen JN, Jepsen SB, Hansen JB, Christensen EF. Implementing a nationwide criteria-based emergency medical dispatch system: a register-based follow-up study. Scand J Trauma Resusc Emerg Med. 2013;21:53.

9. Zakariassen E, Burman RA, Hunskaar S. The epidemiology of medical emergency contacts outside hospitals in Norway - a prospective population based study. Scand J Trauma Resusc Emerg Med. 2010;18:9.

10. Stipulante S, Tubes R, El Fassi M, Donneau AF, Van Troyen B, Hartstein G, et al. Implementation of the ALERT algorithm, a new dispatcher-assisted telephone cardiopulmonary resuscitation protocol, in non-advanced medical priority dispatch system (AMPDS) emergency medical services centres. Resuscitation. 2014;85:177-81.

11. Dami F, Rossetti AO, Fuchs V, Yersin B, Hugli O. Proportion of out-of-hospital adult non-traumatic cardiac or respiratory arrest among calls for seizure. Emerg Med J. 2012;29:758-60.

12. Adnet F, Lapostolle F. International EMS systems: France. Resuscitation. 2004:63:7-9.

13. Dami F, Fuchs V, Praz L, Vader JP. Introducing systematic dispatcher-assisted cardiopulmonary resuscitation (telephone-CPR) in a non-advanced medical priority dispatch system (AMPDS): implementation process and costs. Resuscitation. 2010;81(7):848-52.

14. Official Swiss dispositions for medical dispatch, IAS. http://www.ivr-ias.ch/ cms/upload/imgfile1534.pdf, last visited April $14^{\text {th }} 2015$

15. State of Vaud, Paramedics' protocols [www.bc-cesu.ch/pdf/ AlgoVD_revision2010-11_v6_16.08.12_LPSD.pdf]

16. Tryba M, Brüggemann H, Echtermeyer V. Klassifizierung von erkrankungen und verletzungen im notarztrettungssystemen. Notfallmedizin. 1980;6:725-7.

17. Weiss M, Bernoulli L, Zollinger A. The NACA scale. Construct and predictive validity of the NACA scale for prehospital severity rating in trauma patients. Anaesthesist. 2001;50:150-4.

18. Sefrin P, Sellner J. Qualitätssicherung in der präklinischen Notfallmedizin. Notfallmedizin. 1993;19:267-74.

19. Bonatti J, Göschl O, Larcher P, Wödlinger R, Flora G. Predictors of short-term survival after helicopter rescue. Resuscitation. 1995;30:133-40.

20. American College of Surgeons-COT. Resources for optimal care of injured patient. Chicago: American College of Surgeons-COT; 2006.

21. Mann NC, Schmidt TA, Cone DC. Defining research criteria to characterize medical necessity in emergency medical services: a consensus among experts at the Neely Conference. Prehosp Emerg Care. 2004;8:138-53.

22. Hettinger AZ, Cushman JT, Shah MN, Noyes K. Emergency medical dispatch codes association with emergency department outcomes. Prehosp Emerg Care. 2013;17:29-37.

23. Khorram-Manesh A, Montán KL, Hedelin A, Kihlgren M, Örtenwall P. Prehospital triage, discrepancy in priority-setting between emergency medical dispatch centre and ambulance crews. Eur J Trauma Emerg Surg. 2011;37:73-8.

24. Scott $G$. The need for 911 research and its impact on operations. Ann Emerg Disp Resp. 2013;1:16-7.

25. Eisenberg MS, Bergner L, Hallstrom A. Cardiac resuscitation in the community. Importance of rapid provision and implications for program planning. JAMA. 1979;241:1905-7.

26. Wilson S, Cooke M, Morrell R, Bridge P, Allan T, Emergency Medicine Research Group (EMeRG). A systematic review of the evidence supporting the use of priority dispatch of emergency ambulances. Prehosp Emerg Care. 2002;6:42-9.

27. Pons PT, Haukoos JS, Bludworth W, Cribley T, Pons KA, Markovchick VJ. Paramedic response time: does it affect patient survival? Acad Emerg Med. 2005;12:594-600.

28. Scerbo M, Radhakrishnan H, Cotton B, Dua A, Del Junco D, Wade C, et al. Prehospital triage of trauma patients using the random forest computer algorithm. J Surg Res. 2014;187:371-6.

29. Ohshige K, Kawakami C, Mizushima S, Moriwaki Y, Suzuki N. Evaluation of an algorithm for estimating a patient's life threat risk from an ambulance call. BMC Emerg Med. 2009;9:21.

30. Schlechtriemen T, Burghofer K, Lackner CK, Altemeyer KH. Validierung des NACA-Scoreanhand objektivierbarer Parameter. Notfall\&Rettungsmedizin. 2005;8:96-108

31. Knapp J, Bernhard M, Hainer C, Sikinger M, Brenner T, Schlechtriemen T, et al. Is there an association between the rating of illness and injury severity and the experience of emergency medical physicians? Anaesthesist. 2008;57:1069-74 
32. Lindström V, Karlsten R, Falk A-C, Castrèn M. Feasibility of a computer-assisted feedback system between dispatch centre and ambulances. Eur J Emerg Med. 2011;18:143-1.

33. Ek B, Edstrom P, Toutin, Svedlund M. Reliability of a Swedish pre-hospital dispatch system in prioritizing patients. Int Emergy Nurs. 2013;21:143-9.

34. Lu TC, Chen YT, Chow-In Ko P, Lin CH, Shih FY, Yen ZS, et al. The demand for prehospital advanced life support and the appropriateness of dispatch in Taipei. Resuscitation. 2006;71:171-9.

35. Sporer KA, Youngblood GM, Rodriguez RM. The ability of emergency medical dispatch codes of medical complaints to predict ALS prehospital interventions. Prehosp Emerg Care. 2001;11:192-8.

36. Neely KW, Eldurkar JA, Drake ME. Do emergency medical services dispatch nature and severity codes agree with paramedic field findings? Acad Emerg Med. 2000;7:174-80.

\section{Submit your next manuscript to BioMed Central and take full advantage of:}

- Convenient online submission

- Thorough peer review

- No space constraints or color figure charges

- Immediate publication on acceptance

- Inclusion in PubMed, CAS, Scopus and Google Scholar

- Research which is freely available for redistribution 